\title{
Short communication: Characterization of an atypical maltose-negative Staphylococcus aureus through the use of phenotypic and molecular techniques
}

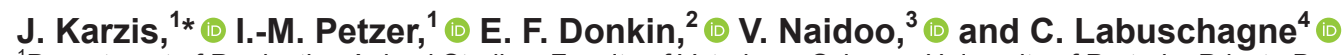 \\ ${ }^{1}$ Department of Production Animal Studies, Faculty of Veterinary Science, University of Pretoria, Private Bag X04, Onderstepoort 0110, \\ South Africa \\ ${ }^{2}$ Department of Animal and Wildlife Sciences, University of Pretoria, Private Bag X20, Hatfield 0028, South Africa \\ ${ }^{3}$ Department of Paraclinical Sciences, Faculty of Veterinary Science, University of Pretoria, Private Bag X04, Onderstepoort 0110 , South Africa \\ ${ }^{4}$ Inqaba Biotechnical Industries (Pty) Ltd., PO Box 14356, Hatfield 0028, South Africa
}

\begin{abstract}
The most clinically relevant staphylococci in veterinary medicine are those that are coagulase-positive, namely Staphylococcus aureus. During microbiological udder health monitoring (2009-2018), a new S. aureus strain (coagulase-positive and maltose-negative) was discovered as an emerging udder pathogen during routine examinations of South African dairy herds. This study challenged the conventional microbiological diagnosis of staphylococci by comparing its results to those of the MALDI-TOF mass spectrometry and 16S rRNA sequencing. Both of these tests confirmed that the maltose-negative staphylococcus (MNS), identified as Staphylococcus pseudintermedius by conventional microbiology, was S. aureus ST2992. Multi locus sequence typing was performed on 3 of the MNS isolates and indicated that these isolates were of single origin. These strains tested positive for both MALA and MALR genes (control: S. aureus ATCC 25923). Although the $\alpha$-glucosidase gene was present, it was not expressed phenotypically. The latter may be attributed to the abnormal stop codon identified in the MALA gene sequence of $S$. aureus ST2992 (GenBank accession number, MN531305). The newly identified MNS has a field behavior different to that of maltose-positive $S$. aureus, and more similar to the low virulence of non-aureus staphylococci.
\end{abstract}

Key words: Staphylococcus aureus, maltose-negative, molecular identification, abnormal stop codon

\section{Short Communication}

Staphylococcus aureus remains a leading cause of IMI in dairy cows (Tenhagen et al., 2009). Petzer et

Received November 12, 2019.

Accepted April 1, 2020.

*Corresponding author: joanne.karzis@up.ac.za al. (2017) reported that $9.05 \%$ of IMI were caused by S. aureus in South African dairy herds (2001-2015), and additional studies have reported 10 to $40 \%$ in other countries (Basanisi et al., 2017; Liu et al., 2017). More recently, isolates that were all maltose-negative, and formerly identified as Staphylococcus intermedius by phenotypic characteristics, were reclassified into 3 clusters-Staphylococcus intermedius, Staphylococcus pseudintermedius (not yet characterized in dairy cattle), and Staphylococcus delphini-based on the nucleotide sequence analysis of the SODA and HSP60 genes (Sasaki et al., 2007).

The main objective of this study was to assess the adequacy of the conventional microbiological identification method. The initial hypothesis tested was to confirm the identity of this maltose-negative Staphylococcus (MNS) species.

Milk samples received for routine udder health investigations, taken from all lactating cows in dairy herds, were analyzed using conventional microbiological methods. Thirty-one coagulase-positive, MNS isolates that originated from 10 out of a total of 880 farms in 4 of the 9 provinces of South Africa were examined (2009-2018). These 31 isolates all originated from subclinical mastitis cases.

The conventional microbiological identification (on tryptose blood agar) was done at the Milk Laboratory (University of Pretoria, Onderstepoort, South Africa) according to the methods recommended by the National Mastitis Council, as in Petzer et al. (2017). The catalase, coagulase (Staphylase test, Oxoid; Quantum Biotechnologies [Pty] Ltd., Ferndale, South Africa), and maltose agar tests (Merck NT Laboratory Supplies, Halfway House, South Africa) were used for identification of staphylococci (Jorgensen at al., 2015; Petzer et al., 2017). The MALDI-TOF MS identification of these staphylococci was carried out at the National Health Laboratory Service - National Institute for Communicable Diseases (Sandringham, South Africa) using the 
direct transfer method with MALDI Biolayer 3.0 software (Bruker Daltonics, Bremen, Germany; Van Dyk et al., 2016). These isolates were further identified by $16 \mathrm{~S}$ rRNA Sanger sequencing analysis conducted at Inqaba Biotec (Pretoria, South Africa), amplified as previously described (Weisburg et al., 1991). In this study the JO-MALAR-F (5'-CTATAGGTCTACAAATGGCC-3') and JO-MALAR-R (5'-CAGGAGGTGATTAAATGGTTAC-3') primers were used to amplify the $M A L A$ and MALR gene targets. The Jo-Mal-Int, primer with sequence GATGTAATGACAGCAAC was the internal sequencing primer used.

Three of the 23 maltose-negative $S$. aureus isolates (confirmed by MALDI-TOF MS and 16S), 1 from each 2009, 2013, and 2017, were submitted to Inqaba Biotec for further analysis by utilizing multilocus sequence typing (MLST). The internal fragments of 7 housekeeping genes (ARCC, AROE, GLPF, GMK, $P T A$, TPI, and $Y Q I L)$ were amplified as previously described (Enright et al., 2000) and sequenced by using Brilliant Dye V3.1 (Nimagen B.V., Nijmegen, Netherlands) and an ABI 3500 XL Genetic analyzer with a 50 $\mathrm{cm}$ array and POP-7 (Applied Biosystems, Waltham, MA). The sequence of each locus was compared with allele sequences in the MLST database (http://saureus .mlst.net) to define the allelic profile for each isolate and to assign a sequence type, which identified this strain as S. aureus ST2992. The absence of maltose activity was evaluated by testing for the presence of the $M A L A$ and $M A L R$ genes (Egeter and Brückner, 1995). The sequences of the MALA and MALR genes of $S$. aureus ST2992 were compared with that of the $S$. aureus ATCC BAA-39 (NCBI database, https://www .ncbi.nlm.nih.gov/genbank/), conventionally identified maltose-positive $S$. aureus ATCC 25923 (control), S. pseudintermedius ATCC 49444, and coagulase-negative maltose-negative S. aureus JN 861808 (Johler et al., 2012).

The conventional microbiology method misdiagnosed the 31 MNS samples as S. pseudintermedius. Both the MALDI-TOF MS and 16S identification confirmed that out of the 31 organisms, 23 were S. aureus, 4 Staphylococcus chromogenes, 1 Staphylococcus capitis, and 1 Staphylococcus xylosus. The 2 remaining isolates were identified by $16 \mathrm{~S}$ rRNA sequencing as Staphylococcus saprophyticus and Macrococcus caseolyticus, but the MALDI-TOF could not identify them. A Bacillus subtilis was used as the out-group, while Macrococcus caseolyticus was an inbuilt out-group. Further MLST confirmed that 3 of the coagulase-negative $S$. aureus (from the group of 23 identical $S$. aureus strains) were maltose-negative $S$. aureus ST2992, which belongs to clonal complex 97 and were of single origin isolated over time.
The MALA and MALR genes were present in the maltose-negative S. aureus ST2992. However, a stop codon was discovered at base pair 844 of the MALA gene caused by a cytosine to thymine transition, which may result in a truncated $\alpha$-glucosidase protein. Nucleotide sequences of the gene encoding $\alpha$-glucosidase (MALA) that showed the stop codon (Figure 1) and its upstream region in the investigated strain ST2992 have been deposited in GenBank (accession number MN531305).

Both the MALDI-TOF MS and 16S rRNA sequencing tests identified 23 of the total 31 isolates as $S$. aureus. The maltose-negative $S$. aureus ST2992 strain (Schmidt et al., 2017) (classification based on MLST) was shown to be an identical strain of single origin, isolated repeatedly over time. The latter isolates have been shown to belong to the common bovine lineage CC97 (Schmidt et al., 2017). The presence of FEMA gene (Kobayashi et al., 1994) further confirmed that these isolates were not $S$. pseudintermedius, but rather a type of $S$. aureus with an unusual biochemical profile. The MALA and MALR genes were present in both the maltose-negative $S$. aureus ST2992 and the maltosepositive $S$. aureus ATCC 25923 control. The MALA gene encodes an $\alpha-1,4$-glucosidase, or maltase, which also liberates glucose from sucrose. Additionally, it is assumed that the MALR gene participates in the regulation of the gene for maltose transport, and would thus be needed for the full expression of these genes (Egeter and Brückner, 1995). The inactivation of $M A L A$ in a sucrose-6-phosphate hydrolase-deficient Staphylococcus xylosus strain has been shown to result in the complete loss of the residual sucrose hydrolase activity (Egeter and Brückner, 1995).

This study discovered a stop codon at base pair 844 of the MALA gene where there was a cytosine to thymine transition, which resulted in the early termination of the $\alpha$-glucosidase protein. This truncated protein is considered to be the most likely cause of the maltosenegative phenotype of $S$. aureus ST2992. The MALR gene of the maltose-negative $S$. aureus ST2992 was identical to that of the ATCC strain used. An [coagulase-negative and MNS] isolate from the Netherlands did not show the same mutations in the MALA gene sequence as those of the maltose-positive control strains (Johler et al., 2012). Thus, the maltose-negative phenotype is likely caused by various differences occurring in either the MALA or MALR genes, or in both. Further research is needed to explore these differences.

Although conventional microbiology methods are not entirely correct in the identification of coagulasepositive and MNS phenotypically, it is important to differentiate between maltose-negative and positive $S$. aureus isolates during routine diagnosis of mastitis pathogens because these organisms react differently in 


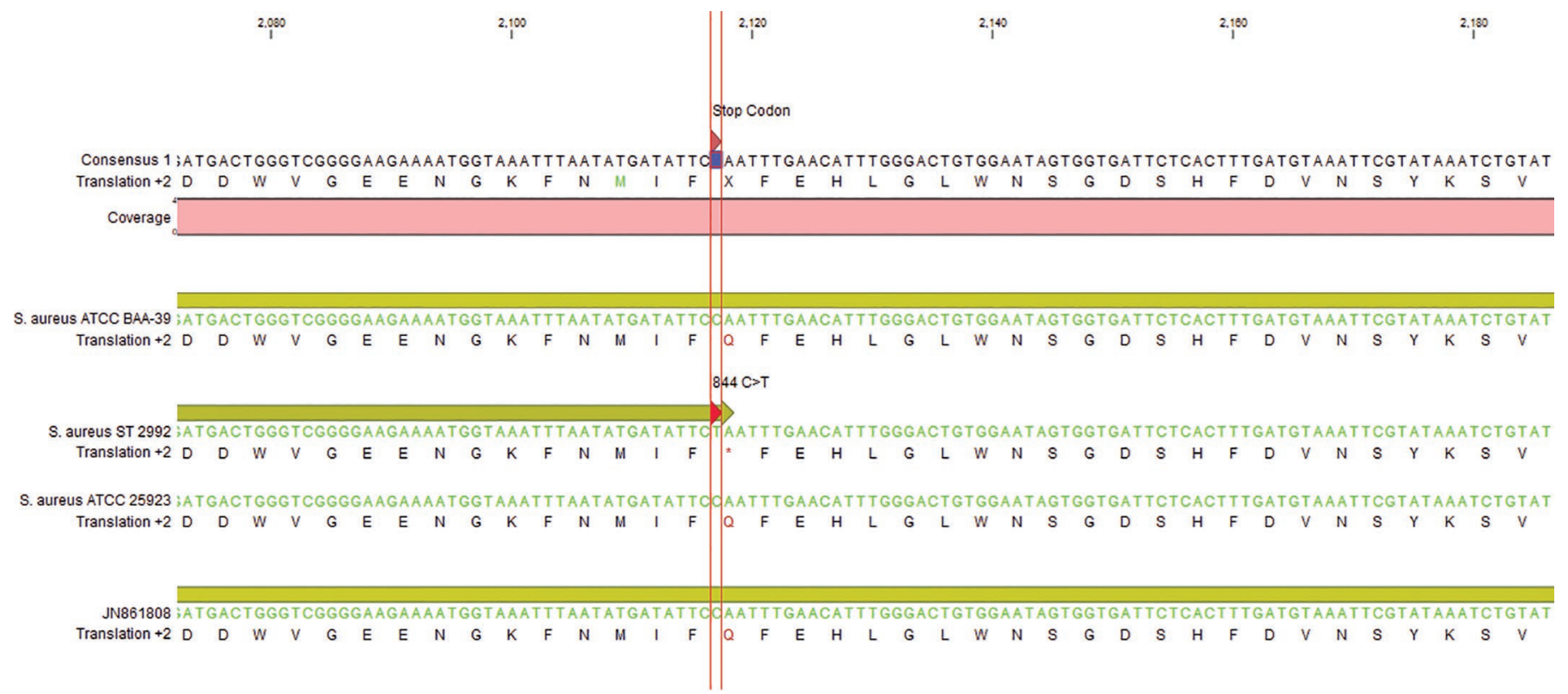

Figure 1. Comparison of the alignment of the MALA gene of the maltose-negative Staphylococcus aureus ST2992 with that of the Staphylococcus aureus ATCC BAA-39 (NCBI database), conventionally identified maltose-positive Staphylococcus aureus ATCC 25923, and coagulase-negative maltose-negative Staphylococcus aureus JN 861808 (Johler et al., 2012), highlighting the mutation at base pair 844 that resulted in the stop codon.

practice. Petzer et al. (2017) showed that $46.74 \%(\mathrm{n}=$ 445) of quarter milk samples with tentatively identified S. pseudintermedius had SCC $\leq 200,000$ cells/mL and $44.31 \%(\mathrm{n}=1,458)$ of composite cow milk samples had SCC $\leq 150,000$ cells $/ \mathrm{mL}$ in similar samples, compared with $11.2 \%(\mathrm{n}=6,087)$ and $17.3 \%(\mathrm{n}=13,522)$ for $S$. aureus (maltose-positive).

Maltose-negative staphylococci represented $1.46 \%$ of bacteria isolated from quarter milk samples (2009 2018). Maltose-negative staphylococci have not been isolated from cows more than once, despite repeated testing, nor could udder parenchyma damage be palpated in infected quarters (Petzer et al., 2017). The latter is in sharp contradiction to the prevalence of maltose-positive $S$. aureus, which causes chronic infections and parenchyma damage (Pyörälä and Pyörälä, 1997; Tenhagen et al., 2009). Different levels of virulence (contagiousness and pathogenicity) have also been widely described among typical $S$. aureus (Cremonesi et al., 2015; Leuenberger et al., 2019). Thus, the maltose-negative $S$. aureus ST2992 could be one of the many genotypes of $S$. aureus not contagious and with "environmental" behavior similar to that of non-aureus staphylococci (Pyörälä and Taponen, 2009).

The strength of this study lies in proving that the maltose-negative $S$. aureus ST2992 with the abnormal stop codon is different from the conventionally identified maltose-positive $S$. aureus, and that the MNS were of single origin.

\section{ACKNOWLEDGMENTS}

The authors thank the producers for allowing them to use data and samples obtained on their farms, the staff of the Milk Laboratory, Department of Production Animal Studies, Faculty of Veterinary Science, Onderstepoort, for the laboratory analyses: J. C. Watermeyer, R. Badenhorst, E. F. Konaite, K. S. Malekane, N. I. Memma, and O. P. Nchako. The authors also thank Olga Perovic and Marshagne Smith of National Health Laboratory Services (NHLS) - National Institute for Communicable Diseases (NICD; Sandringham, South Africa) for the MALDI-TOF MS analysis. This research was partly funded by the National Research Foundation (Pretoria, South Africa). The authors have not stated any conflicts of interest.

\section{REFERENCES}

Basanisi, M. G., G. La Bella, G. Nobili, I. Franconieri, and G. La Salandra. 2017. Genotyping of methicillin-resistant Staphylococcus aureus (MRSA) isolated from milk and dairy products in South Italy. Food Microbiol. 62:141-146. https://doi.org/10.1016/j.fm 2016.10.020.

Cremonesi, P., F. Pozzi, M. Raschetti, G. Bignoli, E. Capra, H. U. Graber, F. Vezzoli, R. Piccinini, B. Bertasi, S. Biffani, B. Castiglioni, and M. Luini. 2015. Genomic characteristics of Staphylococcus aureus strains associated with high within-herd prevalence of intramammary infections in dairy cows. J. Dairy Sci. 98:68286838. https://doi.org/10.3168/jds.2014-9074.

Egeter, O., and R. Brückner. 1995. Characterization of a genetic locus essential for maltose-maltotriose utilization in Staphylococcus xylo- 
sus. J. Bacteriol. 177:2408-2415. https://doi.org/10.1128/JB.177 9.2408-2415.1995.

Enright, M. C., N. P. Day, C. E. Davies, S. J. Peacock, and B. G. Spratt. 2000. Multilocus sequence typing for characterization of methicillin-resistant and methicillin-susceptible clones of Staphylococcus aureus. J. Clin. Microbiol. 38:1008-1015. https://doi.org/10 .1128/JCM.38.3.1008-1015.2000.

Johler, S., M. Moser, C. Engl, T. Tasara, S. Corti, J. Chen, and R. Stephan. 2012. A coagulase- and $\alpha$-glucosidase negative variant of Staphylococcus aureus: A challenge for routine microbiological diagnostics. J. Clin. Microbiol. 50:1827-1828. https://doi.org/10 .1128/JCM.06345-11.

Jorgensen, J. H., M. A. Pfaller, M. L. Landry, S. S. Richter, D. W. Warnock, K. C. Carrol, and G. Funke. 2015. Chapter 21. Pages 354-406 in Manual of Clinical Microbiology, 11th ed. Vol. 1. J. H. Jorgensen, M. A. Pfaller, M. L. Landry, S. S. Richter, D. W. Warnock, K. C. Carrol and G. Funke, ed. ASM Press, Washington, DC.

Kobayashi, N., H. Wu, K. Kojima, K. Taniguchi, S. Urasawa, N. Uehara, Y. Omizu, Y. Kishi, A. Yagihashi, and I. Kurokawa. 1994. Detection of mecA, femA, and femB genes in clinical strains of staphylococci using polymerase chain reaction. Epidemiol. Infect. 113:259-266. https://doi.org/10.1017/S0950268800051682.

Leuenberger, A., C. Sartori, R. Boss, G. Resch, F. Oechslin, A. Steiner, P. Moreillon, and H. U. Graber. 2019. Genotypes of Staphylococcus aureus: On-farm epidemiology and the consequences for prevention of intramammary infections. J. Dairy Sci. 102:3295-3309. https:// doi.org/10.3168/jds.2018-15181.

Liu, H., S. Li, L. Meng, L. Dong, S. Zhao, X. Lan, J. Wang, and N. Zheng. 2017. Prevalence, antimicrobial susceptibility, and molecular characterization of Staphylococcus aureus isolated from dairy herds in northern China. J. Dairy Sci. 100:8796-8803. https://doi .org/10.3168/jds.2017-13370.

Petzer, I. M., J. Karzis, E. F. Donkin, E. C. Webb, and E. M. C. Etter. 2017. Validity of somatic cell count as indicator of pathogenspecific intramammary infection. J S Afr Vet Assoc 88:1465. https: //doi.org/10.4102/jsava.v88i0.1465.

Pyörälä, S., and E. Pyörälä. 1997. Accuracy of methods using somatic cell count and N-acetyl-beta-D-glucosaminidase activity in milk to assess the bacteriological cure of bovine clinical mastitis. J. Dairy Sci. 80:2820-2825. https://doi.org/10.3168/jds.S0022 -0302(97)76246-5.

Pyörälä, S., and S. Taponen. 2009. Coagulase-negative staphylococci emerging mastitis pathogens. Vet. Microbiol. 134:3-8. https://doi .org/10.1016/j.vetmic.2008.09.015.

Sasaki, T., K. Kikuchi, Y. Tanaka, N. Takahashi, S. Kamata, and K. Hiramatsu. 2007. Reclassification of phenotypically-identified Staphylococcus intermedius strains. J. Clin. Microbiol. 45:27702778. https://doi.org/10.1128/JCM.00360-07.

Schmidt, T., M. M. Kock, and M. M. Ehlers. 2017. Molecular characterization of Staphylococcus aureus isolated from bovine mastitis and close human contacts in South African dairy herds: Genetic diversity and inter-species host transmission. Front. Microbiol. 8:511-15. https://doi.org/10.3389/fmicb.2017.00511.

Tenhagen, B. A., I. Hansen, A. Reinecke, and W. Heuwieser. 2009. Prevalence of pathogens in milk samples of dairy cows with clinical mastitis and in heifers at first parturition. J. Dairy Res. 76:179187. https://doi.org/10.1017/S0022029908003786.

Van Dyk, B. N. W. De Bruin, E. M. Du Plessis, and L. Korsten. 2016. Microbiological food safety status of commercially produced tomatoes from production to marketing. J. Food Prot. 79:392-406. https://doi.org/10.4315/0362-028X.JFP-15-300.

Weisburg, W. G., S. M. Barns, D. A. Pelletier, and D. J. Lane. 1991. $16 \mathrm{~S}$ ribosomal DNA amplification for phylogenetic study. J. Bacteriol. 173:697-703. https://doi.org/10.1128/JB.173.2.697-703.1991.

\section{ORCIDS}

J. Karzis (ㄴ) https://orcid.org/0000-0002-6210-0985

I.-M. Petzer ๑ https://orcid.org/0000-0001-9890-8997

E. F. Donkin (๑) https://orcid.org/0000-0001-6643-0271

V. Naidoo ๑ https://orcid.org/0000-0003-2740-5983

C. Labuschagne $\odot$ https://orcid.org/0000-0002-2771-4922 